\title{
Correction to: Bridging the science-practice gaps in nature-based solutions: A riverfront planning in China
}

\author{
Zhifang Wang $\mathbb{D}^{\mathrm{D}}$, Liyun Huang, Min Xu, Sirui Wang
}

Published online: 24 June 2021

\section{Correction to: Ambio}

https://doi.org/10.1007/s13280-020-01445-2

In the original publication, the given names and surnames of the authors were swapped in one of the references and hence incorrectly cited as Blal et al. (2017) in the article.

The correct reference is provided below.

Adem Esmail, B., D. Geneletti, and C. Albert. 2017. Boundary work for implementing adaptive management: A water sector application. Science of the Total Environment 593: 274-285. https://doi.org/10.1016/j.scitotenv.2017.03. 121

The original article has been corrected.

Publisher's Note Springer Nature remains neutral with regard to jurisdictional claims in published maps and institutional affiliations.

\section{Zhifang Wang $(\bowtie)$}

Address: College of Architecture and Landscape of Peking University, No. 5 Yiheyuan Road Haidian, District, Beijing 100871, People's Republic of China.

e-mail: zhifangw@pku.edu.cn

\section{Liyun Huang}

Address: College of Architecture and Landscape of Peking University, No. 5 Yiheyuan Road Haidian, District, Beijing 100871, People's Republic of China.

e-mail: 1801214244@pku.edu.cn

\section{Min Xu}

Address: College of Architecture and Landscape of Peking University, No. 5 Yiheyuan Road Haidian, District, Beijing 100871, People's Republic of China.

e-mail: xumin7105@pku.edu.cn

\section{Sirui Wang}

Address: College of Architecture and Landscape of Peking University, No. 5 Yiheyuan Road Haidian, District, Beijing 100871, People's Republic of China.

e-mail: 1701214569@pku.edu.cn

The original article can be found online at https://doi.org/10.1007/ s13280-020-01445-2. 